REDES CORPORATIVAS

INMERSIVAS

\section{Nuevas formas de uso de las redes sociales}

\section{Jorge E. Giménez Hernando \\ Coordinador de proyectos}

Desarrollo de Proyectos e Innovación Pedagógica. Instituto de Formación Online, c/ Río Guadalquivir no 13, Urbanización Parque Boadilla, 28660 Boadilla del Monte, Madrid (España) - Email: jgimenez@ifoline.net

\section{Virginia Vázquez Vázquez}

\section{BCS Consulting}

Everis. Av. Manoteras 52, 28050 Madrid (España) - Email: virvazquez@gmail.com

\section{Resumen}

Las redes sociales se están imponiendo como forma de comunicación a través de Internet sobre cualesquiera otros formatos tradicionales, llegando incluso a eclipsar el más reciente y universal correo electrónico. De entre sus múltiples aplicaciones, destacan desde el punto de vista empresarial las redes sociales corporativas, que se conciben como un vehículo extraordinariamente eficaz para transferir el conocimiento que aporte a la compañía ventaja competitiva para posicionarse y operar en el mercado.

Por otra parte, el auge del desarrollo de las nuevas tecnologías basadas en $3 \mathrm{D}$, los mundos virtuales o la realidad aumentada, que se ha
Palabras clave

Mundo virtual, red corporativa, red social, e-Learning, formación, nuevas tecnologías, Web 2.0

Key Words

Virtual world, corporate network, social network, e-Learning, training, new technologies, Web 2.0

\section{Abstract}

The social networks are imposed as form of communication across

Internet on any other traditional formats, managing to eclipse even the most recent and universal e-mail. Among his multiple applications, stand out from the business point of view, the social corporate networks, which are conceived as an extraordinarily effective vehicle to transfer the knowledge that contributes to the company competitive advantage to position and operate on the market. On the other hand, the summit of the development of the new technologies based in 3D, the virtual worlds or the augmented reality, which has been based in the last years on playful and scattering environments, begins to be applied to another kind of contents, trying to take advantage of his realism and motivation.

The present article presents the accomplishment of a pilot experience

in which both concepts have combined, applying the technologies of virtual worlds to the social corporate networks. 
basado en los últimos años en entornos lúdicos y de esparcimiento, está comenzando a aplicarse a otro tipo de contenidos, que se intentan aprovechar de su realismo y motivación.

El presente artículo presenta la realización de una experiencia piloto en que se han aunado ambos conceptos, aplicando las tecnologías de mundos virtuales a las redes sociales corporativas, y permitiendo la comparación de los resultados obtenidos con los acumulados en experiencias anteriores de acceso web tradicional. Dicha comparación se ha basado en las encuestas realizadas a los usuarios tras la experiencia piloto.

\section{Introducción}

Las redes sociales se están imponiendo como forma de comunicación a través de Internet sobre cualesquiera otros formatos tradicionales, llegando incluso a eclipsar el más reciente y universal correo electrónico. Inicialmente, las aplicaciones más extendidas han ido encaminadas a las relaciones personales, diversificándose actualmente según numerosos y diversos objetivos. De entre estas múltiples aplicaciones, destacan desde el punto de vista empresarial las redes sociales corporativas, que "se conciben como un vehículo extraordinariamente eficaz para transferir, formal o informalmente, ese conocimiento que aporte a la compañía ventaja competitiva para posicionarse y operar en el mercado sin peligro de que esta información sensible 'traspase' las fronteras invisibles de la organización"i.

Por otra parte, las tecnologías multimedia basadas en 3D, como los mundos virtuales, encaminados inicialmente al entretenimiento de los videojuegos, también están dando paso a nuevas aplicaciones. El desarrollo de simuladores permite la formación de profesionales en entornos en los que un prototipo real resulta difícil de desarrollar o muy caro, estando su aplicación cada vez más extendida a entornos muy diversos. Se intenta aprovechar en todos esos casos el realismo y motivación inherente a los mundos virtuales, además de la cantidad cada vez mayor de usuarios "nativos digitales", que asumen su uso de una forma natural.

Teniendo en cuenta ambas circunstancias, así como la extensa experiencia previa en ambos entornos, el Instituto de Formación Online (IFO), empresa pionera en el sector y con un fuerte carácter innovador, ha abordado una experiencia piloto en la que se aúnan ambos conceptos: el acceso y utilización de las redes sociales corporativas mediante una interfaz de tipo mundo virtual, en particular con la metodología playLearning ${ }^{\circledR}$ desarrollada por IFO ${ }^{\mathrm{ii}}$.

El presente artículo presenta la realización de dicha experiencia piloto en la Red de Expertos Iberoamericanos (REI) en e-Learning de la Fundación CEDDET ${ }^{\mathrm{iii}}$, permitiendo la comparación de los resultados obtenidos con los acumulados en experien- 
cias anteriores de acceso web tradicional. La comparación se ha realizado en base a las encuestas rellenadas por los usuarios al finalizar la experiencia piloto.

\section{Objetivos}

Los objetivos de la experiencia piloto desarrollada, en la que se han aunado las redes sociales corporativas, con las tecnologías de mundos virtuales 3D son:

- Comparar los resultados obtenidos a partir de las encuestas rellenadas por los usuarios, mediante el acceso web estándar y a través de la metodología de

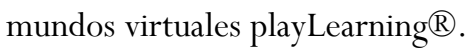

- Validar la metodología playLearning® como tipo de acceso a las redes sociales.

- Encontrar posibles vías de mejora y nuevas aplicaciones en base a las opiniones de los usuarios.

\section{Metodología}

La metodología utilizada se ha basado en la aplicación de una interfaz de acceso me-

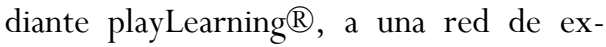
pertos en e-Learning, seleccionando un conjunto de funcionalidades de la red original, que han sido remodeladas con un aspecto 3D, dentro de un escenario de tipo mundo virtual, que ha posibilitado además añadir nuevas herramientas no disponibles en el tipo de acceso original de tipo web estándar.
Se ha implementado un itinerario de actividades, de forma que los usuarios invitados a la experiencia han debido realizarlas secuencialmente durante la experiencia piloto.

Los resultados se han medido a partir de las encuestas rellenadas por los usuarios al finalizar el itinerario.

\section{Antecedentes}

La experiencia descrita en el presente artículo, ha podido ser llevada a cabo gracias a la experiencia previa en la implantación y dinamización de redes, en el desarrollo de materiales formativos con formato de mundo virtual de carácter inmersivo, y al deseo de innovación y mejora.

\subsection{Experiencia en la implantación y dinamización de redes}

El Instituto de Formación Online ha desarrollado un conjunto de proyectos de 
implantación y dinamización de redes, entre los que destacan:

- FUNDACIÓN CEDDET. El Programa Redes de Expertos Iberoamericanos (REI) es un proyecto que nació a finales de 2004 con el objetivo de que profesionales iberoamericanos de distintas áreas temáticas se mantuvieran en contacto y continuaran intercambiando experiencias y conocimientos, con el fin último de contribuir al fortalecimiento institucional de sus países. Las Redes de Expertos Iberoamericanos, auspiciadas por instituciones españolas e internacionales, son comunidades virtuales en las que se promueve el intercambio de conocimientos y mejores prácticas, así como la colaboración entre organizaciones públicas en el espacio iberoamericano para el desarrollo de actividades de gestión del conocimiento, esencialmente mediante la formación continua, celebración de Foros de Expertos, elaboración de Revistas digitales, o generación de bibliotecas temáticas, entre otras. Actualmente el Programa cuenta con 15 redes en funcionamiento.

- CEPSA. Gestión integral de los entornos colaborativos consistentes en comunidades de profesionales de la empresa que participan en grupos de mejora sobre problemáticas vinculadas a las competencias de la organización. La metodología de trabajo se compone de una red virtual sobre plataforma Moodle, sesiones presenciales periódi- cas y trabajos de autoformación aprovechando los recursos generados en las propias redes.

- GARRIGUES. CENTRO DE ESTUDIOS. Gestión del Programa de Integración para los nuevos profesionales de la firma. La metodología combina formación online con sesiones presenciales. La comunidad virtual creada pone en contacto a los nuevos profesionales de la firma en toda España, ofrece información sobre el trascurso del programa integral y mantiene el vínculo entre expertos y destinatarios. En la plataforma Moodle de IFO los destinatarios realizan actividades, proyectos $\mathrm{y}$ participan en foros.

- FOREM CONFEDERAL. Se está dotando a la institución de un entorno de red profesional que cumpla una triple funcionalidad: disponer de un área de trabajo compartido para la propia institución con acceso restrictivo, un entorno abierto a la colaboración con otros FOREMs Autonómicos y por último, un espacio de difusión e información de las acciones y actividades de FOREM Confederal.

AEVAL. Se ha desarrollado un espacio de red formativa que aglutine a los participantes que han recibido una formación muy específica sobre una temática y se les ofrece este soporte para que puedan mantener contacto, compartir sus experiencias y profundizar en el área en el que son especialistas. 


\subsection{Experiencia en el desarrollo de materiales formativos con formato de mundo virtual de carácter inmersivo}

IFO comenzó el desarrollo de la tecnología playLearning ${ }^{\circledR}$ en 2006 a raíz del proyecto "Gestión de Residuos Radiactivos" para ENRESA, con el objetivo de difundir entre los jóvenes un conocimiento real de la seguridad en el tratamiento y conservación de los residuos radiactivos y del compromiso de ENRESA con el medioambiente. Se desarrolló un programa completo basado en dos escenarios, uno que cubría el currículo de enseñanza secundaria en materia de radiactividad, y otro descriptivo de los protocolos de gestión de los residuos radiactivos en España.

Tras el éxito del proyecto, IFO comenzó el desarrollo de nuevos proyectos con esta innovadora tecnología, dirigidos entonces a la formación en entornos empresariales. Se comenzó con Virtual Coaching, proyecto piloto de un entorno virtual para el aprendizaje, práctica y desarrollo de competencias profesionales relacionadas con trabajos de oficina, en el que el usuario elige un personaje que le representa con el que se va a poder mover por el entorno virtual de oficina, donde se encontrará diversos retos para el desarrollo de sus competencias profesionales o habilidades directivas.

Más tarde, en el marco del proyecto Comenius Virtual, se han desarrollado:
- Una herramienta de generación de mundos virtuales. Esta utilidad permite construir de forma rápida y sencilla los elementos necesarios para que el usuario puede escoger su avatar y desenvolverse en el mundo virtual para tener acceso a diversos contenidos digitales y recursos multimedia para su formación, que también son desarrollados con esta herramienta.

- Tres prototipos de mundos virtuales:

1. English World para la práctica de inglés para estudiantes de dirección de empresas de la escuela de negocios EADA.

2. Tecnología Sanitaria para la práctica del manejo de Ecógrafos en el área de salud, en colaboración con ISD.

3. Red Social para el acceso a redes sociales y profesionales de carácter internacional, con aplicación a la Red de Expertos Iberoamericanos en eLearning de la Fundación CEDDET (el objeto del presente artículo).

- Se han puesto en marcha tres "Living labs" como pruebas piloto de carácter internacional destinadas a validar la extensión de cada uno de los prototipos.

Comenius Virtual es un proyecto de investigación desarrollado con un plan Avanza I+D del Ministerio de Industria, y cofinanciado por el Fondo Europeo de Desarrollo Regional (FEDER). 
Además, se ha desarrollado un proyecto para la práctica de los protocolos de la Higiene de manos en el mundo sanitario para la Fundación para la Investigación Biomédica del Hospital Gregorio Marañón.

Dentro del ámbito de la tecnología sanitaria, se están desarrollando además actualmente los contenidos formativos de nuevos aparatos, entre los que se encuentran el electrocardiógrafo, el desfibrilador y el espirómetro.

Por último, dentro del ámbito del proyecto Galeno $\mathrm{XXI}^{\mathrm{iv}}$, se está avanzando en la tecnología de aprendizaje en mundos virtuales inmersivos, desarrollando todo un nuevo concepto de 3D subjetivo.

\subsection{Deseo de innovación y mejora}

El Instituto de Formación Online nace en 1996 dedicado a dar respuesta a las necesidades de las organizaciones mediante soluciones de formación aplicando las Nuevas Tecnologías.

En sus quince años de experiencia, IFO se ha convertido en empresa líder en España referencia en el desarrollo de proyectos globales de e-Learning.
En IFO la investigación y la innovación son una constante que nos permite proporcionar a nuestros clientes las mejores soluciones de formación, tanto desde el punto de vista metodológico como el tecnológico.

Para la persecución de estos objetivos, IFO aborda habitualmente nuevos proyectos en los que pone en práctica nuevas tecnologías y nuevos conceptos en e-Learning, entre los que se encuentra el descrito en el presente artículo.

Teniendo presente todas estas circunstancias, se planteó la siguiente pregunta:

$i^{\text {Los entornos inmersivos pueden }}$ mejorar la interacción y la motivación de los usuarios en las redes corporativas?

Ante la falta de experiencias prácticas en IFO, o de datos obtenidos de otras fuentes, que pudieran dar una respuesta realista a este interrogante, se decidió dar un paso más allá de proyectos anteriores y aunar ambas tecnologías, dando origen a este proyecto. De esta forma se podría comparar los resultados en redes corporativas obtenidos "sin" y "con" el acceso a través de un mundo virtual, obteniendo datos sobre los entornos inmersivos aplicados a las Redes Corporativas. 


\section{Entorno de investigación}

\subsection{Motivos por los que escogimos una red real: comparación de resultados}

Basado en las experiencias desarrolladas en redes sociales, de las que se cuenta con datos cuantitativos, se decidió llevar a cabo una experiencia innovadora, dando acceso a las redes sociales a través de la tecnología playLearning ${ }^{\circledR}$, realizando un prototipo completo, con un conjunto de funcionalidades de la Red de Expertos Iberoamericanos en e-Learning de CEDDET. De esta manera, recogiendo a su vez datos cuantitativos de dicha experiencia, se puede llevar a cabo una comparación de los resultados con/sin entrada playLearning ${ }^{\circledR}$ y así

validar el uso de los mundos virtuales como acceso a las redes sociales.

Además, se diseñó un itinerario de actividades con un subconjunto de todas las funcionalidades disponibles, y se preparó un seguimiento tutorizado de todo ello.

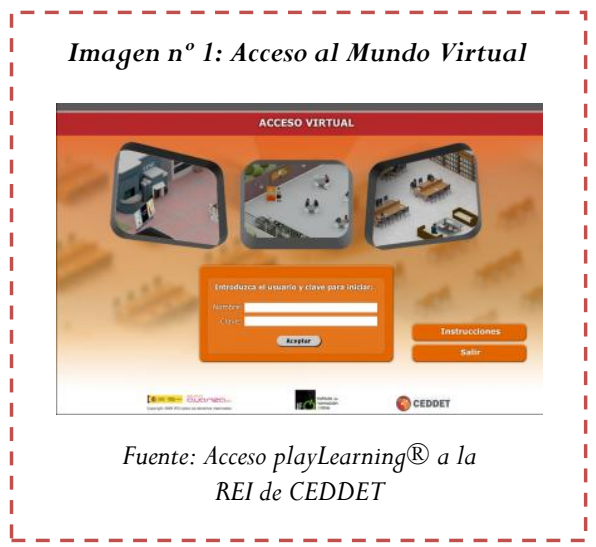

\section{Diseño de la prueba piloto}

\subsection{Solución técnica}

La solución técnica se desarrolló en torno a los siguientes objetivos, coincidiendo con los de la REI de e-Learning:

Mantener el contacto entre los profesionales iberoamericanos del área en que se desarrolla la REI.

- Crear un foro permanente para la difusión de conocimientos, noticias y casos prácticos de organizaciones latinoamericanas.
- Servir como punto de contacto operativo entre profesionales que demanden colaboración de colegas de otros países para el desarrollo de sus tareas.

- Contribuir a la mejora continua de los programas de formación.

Para cumplir con los objetivos del proyecto se desarrolló el mundo virtual, con las siguientes características principales:

- El prototipo de Red Social consiste en una interfaz de acceso a la Red de Ex- 
pertos de CEDDET mediante metodología playLearning ${ }^{\circledR}$.

- El acceso a través del prototipo está previsto como una alternativa experimental al acceso estándar a la Red de Expertos, que se mantiene simultáneamente.

- Su diseño tiene un carácter genérico aplicable a diversas Redes de Expertos, contando además con elementos personalizados a la red de e-Learning.

- El prototipo está destinado fundamentalmente a alumnos que han participado en cursos de CEDDET. Además de los alumnos, los coordinadores pueden acceder con algunos permisos adicionales a los del alumno.

- El usuario realiza una selección del avatar que mejor le represente de entre un conjunto que se le presenta a la entrada a la Red.

- En el inicio se presenta una explicación de las funcionalidades disponibles en el prototipo, así como de uso de la interfaz.

- Además de la interfaz general con metodología playLearning ${ }^{\circledR}$, también es de este tipo cada uno de los recursos de la red a la que se accede.

- Los recursos de la Red a los que da acceso el prototipo se detallan más adelante.
Se seleccionaron además los siguientes perfiles de usuario con acceso a la Red a través del prototipo:

- Usuarios generales: pueden publicar documentación e interactuar con los recursos generales que ofrece el entorno. Los contenidos que se publican no se supervisan previamente, se ofrece la posibilidad de "denunciar" los contenidos inapropiados tras la publicación.

- Usuarios con permisos ampliados: En este grupo incluimos a los coordinadores que pueden acceder a los mismos recursos que el perfil de usuario sumando además algunas aplicaciones específicas, concretamente "Administrar Redes”, "Encuestas”, “Notificaciones”, etc.

- Usuario Coordinador temático, con permisos intermedios entre los dos usuarios anteriores.

Una vez dentro del mundo virtual, en primer lugar, el usuario selecciona el avatar que mejor le represente de entre un conjunto de 12 avatares variado en edades y sexo, que se le presenta a la entrada a la Red.

Tras la selección del avatar, el usuario visualiza un vídeo de introducción cada vez que entre en la Red. Esta presentación se puede "Saltar" para poder acceder sin necesidad de verlo completamente. 
En dicho vídeo se incluye:

- Bienvenida al usuario.

- Presentación de la REI.

- El objetivo principal que ésta persigue.

- Introducción al nuevo acceso.

- Instrucciones de uso.

\subsection{Diseño de actividades en la red}

De entre todas las funcionalidades disponibles en el acceso web estándar a la Red de Expertos de CEDDET, se seleccionó un conjunto representativo, aprovechando las posibilidades de la metodología playLearning® para añadir otras nuevas.

A las primeras se accede mediante puntos de interacción en el escenario, mientras que a las últimas se accede desde el interfaz. Las funcionalidades accesibles desde el escenario son:

- Multiusuario y Comunicación Síncrona: los usuarios de la Red se ven unos a otros cuando están conectados simultáneamente, pudiendo interactuar:

1. Usuario-Usuario: mediante mensajes instantáneos de tipo bocadillo o mediante una ventana en la interfaz.

2. Coordinador-Usuario: si hay algún coordinador conectado se puede reconocer por un indicador al efecto. El coordinador puede lanzar un mensaje colectivo para convocar a una actividad grupal (por ejemplo una reunión en el foro de expertos, o un chat de dudas, etc.) o realizar una explicación que considere necesaria de algún tema.

- Vídeos: los participantes pueden acceder a un recurso específico en el que están publicados distintos vídeos con temáticas variadas relacionadas con la Red de e-Learning. El usuario puede visualizar dichos vídeos y realizar búsquedas entre los recursos publicados.

- Novedades: a través de este apartado pueden acceder a las siguientes opciones: noticias, carrusel de anuncios y revista digital. Se tratan como Novedades los siguientes recursos: Noticias recientes, Plan de Actividades y Destacados. Además de las categorías:

1. Última edición de la Revista.

2. Último boletín.

3. Congresos y Eventos (no los que se han realizado sino los próximos).

- Comunicación: se pueden realizar las siguientes acciones: correo interno, Chat, Blogs, miembros de la REI (incluye buscador) y foros (en todas sus categorías).

- Documentación: A partir del gestor documental, se accede a las siguientes categorías:

1. Documentación de los Cursos.

2. Legislación.

3. Documentación Temática.

4. Experiencias. 


\section{Seminarios.}

En todos los listados, el usuario puede realizar búsquedas, descargar, ver y aportar información.

- Sugerencias: espacio donde aparecen mensajes instantáneos con sugerencias: por ejemplo, "Accede a las novedades", "Participa en el foro de expertos", "Incluye un término en el glosario", etc.

Los recursos accesibles desde la Interfaz son:

- Perfil: ficha con datos sobre el usuario recogidos por la plataforma. Dicho perfil está alimentado por el sistema de registro SGCO (Sistema de Gestión de Cursos Online) mediante una pasarela.

- Barra de participación actual: Indica el nivel de participación del usuario en la REI en el último mes (obtenido de los datos de plataforma). Está vinculado a las actividades que se desarrollan en la REI, por lo que a más participación, mayor será este indicador. Este recurso está relacionado con las Estadísticas.

- Encuesta de valoración del prototipo: enlace "Valoración".

- Ayuda: La ayuda pretende guiar al usuario y tenerle orientado en todo momento:

O Instrucciones: Ayuda general sobre el escenario accesible en todo momento desde el Interfaz.

- Ayuda específica de cada punto de interacción: dentro de cada punto de interacción aparece una Ayuda para que el usuario tenga disponible la información sobre la funcionalidad y manejo de cada apartado de la REI. Una vez se cierre esa ayuda está disponible a través de un icono localizado para que el usuario pueda visualizarla.

- Calendario: contiene los eventos próximos más importantes. Al pulsar sobre cada uno de ellos se abre una ventana con los detalles del evento correspondiente. Esta funcionalidad se muestra mediante un buscador de eventos organizados por categorías (las acordadas y ya existentes en el entorno web estándar). Además permite ordenar por "Eventos próximos", “de todos los usuarios" y de "mis contactos".

- Contactos: enlace que lleva al recurso Contactos de la REI.

- Mensajes: enlace que lleva al recurso Mensajes de la REI.

- Mapa Site: mapa donde el usuario puede verse ubicado mediante un punto, en función del país que tenga configurado en su perfil. Asimismo se pueden ver también como puntos, en qué países se ubican el resto de usuarios de la Red que están conectados.

- El Mapa permite ver los nombres de los usuarios conectados en ese momento.

- GPS: es un mapa esquemático del escenario en el que se puede ver la situación de los puntos de interacción. 
- Escenario: enlace que lleva a un mapa del escenario con todas las zonas calientes marcadas.

- Guardar: permite guardar los pasos realizados.

- Salir: permite salir del prototipo.

- Chat: en relación con la comunicación síncrona. Se incluye un enlace a chat, el cual abre una ventana de conversación donde se puede escoger entre un participante situado en el escenario en ese momento o todos los que estén situados para mantener una conversación.
Tiempo de actividad: marcador con el tiempo de permanencia en el programa.

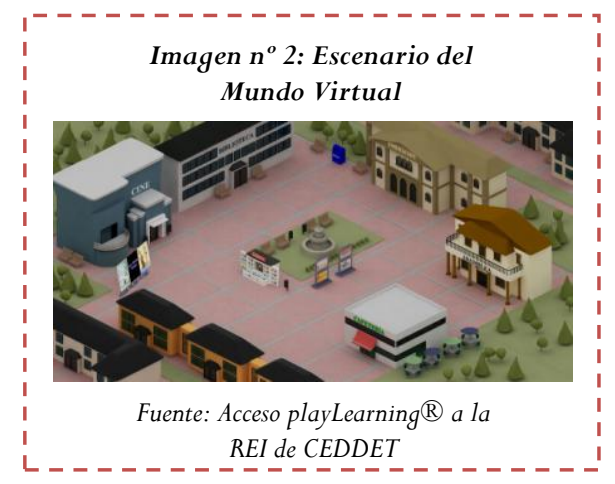

\section{Desarrollo de la prueba piloto}

La prueba piloto se ha desarrollado desde el 12 de julio al 1 de agosto de 2010, con la participación de 100 usuarios de la REI en e-Learning de CEDDET.

\subsection{Actividades realizadas}

De entre todas las funcionalidades incluidas en el mundo virtual, se han seleccionado un subconjunto representativo que los usuarios deben realizar durante el desarrollo de la prueba piloto:

- Actividad 1: Presentación.

Ubicación: Cafetería.

Recurso utilizado: Foro/libre.

Descripción: El usuario acude al edificio Cafetería, donde encuentra el Foro Presentación que ha sido publicado por el Administrador y ha de presentarse.
- Actividad 2: Debate.

Ubicación: Aula de formación.

Recurso utilizado: Grupo.

Descripción: El usuario acude al edificio Aula de formación, donde el Administrador ha incluido un Grupo específico para la realización de este living lab. El usuario accede a dicho Grupo, se descarga el documento que ha publicado el Administrador para realizar esta actividad (pdf que introduce la teoría de los mundos virtuales), y a continuación debe publicar un comentario sobre el documento leído. El comentario que publique el usuario puede ser guiado (partiendo de una pregunta que haya publicado el administrador como primer comentario) o libre. 
- Actividad 3: Cine.

Ubicación: Cine.

Recurso utilizado: Vídeos de la red.

Descripción: El administrador previamente ha embebido una serie de vídeos de Youtube sobre temas relacionados con la temática a tratar (mundos virtuales, playLearning ${ }^{\circledR}$, etc.). El usuario accede al edificio Cine y ve como en la pantalla del cine se le presentan a modo de cartelera los vídeos. La actividad consiste en visualizar dichos vídeos y realizar un comentario en cada uno de ellos. El comentario que publique el usuario puede ser guiado (partiendo de una pregunta que haya publicado el administrador como primer comentario) o libre.

- Actividad 4: Foro.

Ubicación: Asamblea.

Recurso utilizado: Foro temático.

Descripción: Para esta actividad ha participado un experto de CEDDET en contenidos que sirve como profesor para que modere un foro relacionado con la temática del living lab. El experto ha publicado un foro y los usuarios tienen que acudir al edificio Asamblea y realizar sus comentarios. Se propone como temática del foro: Ventajas y desventajas de los entornos virtuales como herramienta educativa. Para terminar el Foro, el experto indica a los participantes la existencia de un chat para aclarar las conclusiones del tema a tratar. El experto indica además la hora del chat y que no es obligatorio pero si interesante (ver Actividad 8).

Actividad 5: Artículo.

Ubicación: Kiosko.

Recurso utilizado: Artículo.

Descripción: la actividad consiste en que el usuario tiene que acceder al kiosko y ha de colgar un artículo de interés sobre un tema a definir. El artículo puede buscarlo de Internet, o puede escribirlo él mismo.

- Actividad 6: Conclusiones.

Ubicación: Biblioteca.

Recurso utilizado: Listado, comentario.

Descripción: el usuario accede al edificio Biblioteca y ha de buscar un documento que el administrador ha publicado previamente donde se explican las conclusiones de los mundos virtuales. El administrador como primer comentario pide a los usuarios que indiquen una única conclusión de lo leído. Una vez que el usuario ha leído dicho documento ha de publicar una conclusión acerca de los mundos virtuales.

Actividad 7: Encuesta.

Ubicación: Interfaz: enlace "Valoración".

Recurso utilizado: ninguno de la REI.

Descripción: el usuario accede al enlace "Valoración", situado en el interfaz del 
prototipo. Le aparece una encuesta de valoración que tiene que rellenar.

Otras actividades:

- Actividad 8: Chat.

Peculiaridad: esta actividad no es obligatoria.

Ubicación: Interfaz: enlace "Chat".

Recurso utilizado: ninguno de la REI.

Descripción: el usuario accede al enlace Chat, situado en el interfaz del prototipo. El experto propuesto por CEDDET modera un chat que trata de llegar a conclusiones sobre el foro realizado en la actividad 4. Esta actividad no es obligatoria ya que por motivos de agenda no podemos asegurar que todos los usuarios puedan participar.

- Actividad 9: Buzón.

Peculiaridad: esta actividad sólo la tiene que realizar el administrador.

Ubicación: Buzón.

Recurso utilizado: Notificaciones.

Descripción: el administrador ha de enviar un documento con las conclusiones generadas sobre el tema general a tratar en el living lab.

\subsection{Participación}

A partir de los datos registrados en la plataforma, se han obtenido los datos de participación, que se detallan en los siguientes Cuadro ${ }^{\circ} 1$ y Gráfico ${ }^{\circ} 1$.
Cuadro $n^{o}$ 1. Datos de participación

\begin{tabular}{|l|l|}
\hline DATOS GENERALES & N $^{\mathbf{0}}$ \\
\hline $\begin{array}{l}\text { Participantes inscritos inicialmente } \\
\text { en la experiencia piloto }\end{array}$ & 100 \\
\hline Bajas & 13 \\
\hline Usuarios que participan & 74 \\
\hline Usuarios que NO participan & 8 \\
\hline Índice de Participación & $74 \%$ \\
\hline
\end{tabular}

Fuente: Elaboración propia a partir de los datos obtenidos en la Red

Gráfico $n^{\circ}$ 1: Índice de Participación

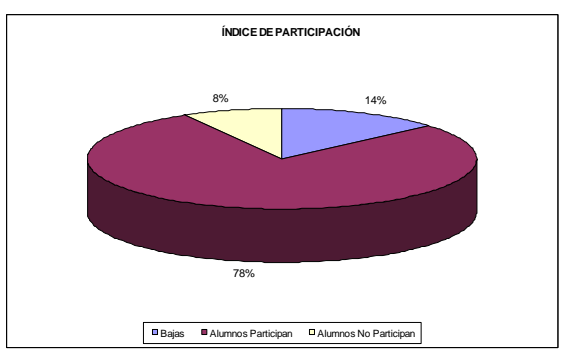

Fuente: Elaboración propia

\subsection{Resultados}

En primer lugar, incluimos los resultados de una encuesta previa, realizada a usuarios de la REI de e-Learning con el acceso web estándar. Para las valoraciones, se ha usado una escala de 1 a 4 , siendo:

- 1: Totalmente en desacuerdo.

- 2: En desacuerdo.

- 3: De acuerdo.

- 4: Totalmente de acuerdo. 
Cuadro $n^{o}$ 2. Resultados mediante acceso web: Valoración global

\begin{tabular}{|l|l|}
\hline VALORACIÓN GLOBAL & $\mathbf{3 . 2 4}$ \\
\hline El uso de la REI es intuitivo y amigable & 3.02 \\
\hline Durante el uso de la REI NO me he encontrado problemas técnicos. & 3.38 \\
\hline $\begin{array}{l}\text { Si he tenido problemas técnicos, me he sentido atendido y la resolución } \\
\text { ha sido rápida y satisfactoria. }\end{array}$ & 3.56 \\
\hline
\end{tabular}

Fuente: Elaboración propia a partir de los datos obtenidos en la Red

A continuación, se encuentran los resultados obtenidos mediante el acceso playLearning ${ }^{\circledR}$, que se han extraído de las encuestas realizadas a los usuarios al finalizar la prueba piloto. Se incluyen la Valoración global de la prueba así como la de la acción tutorial. Igualmente que en el caso ante- rior, para todas las valoraciones se ha usado una escala de 1 a 4 , siendo:

- 1 : Totalmente en desacuerdo.

- 2: En desacuerdo.

- 3: De acuerdo.

- 4: Totalmente de acuerdo.

Cuadro $n^{\circ}$ 3. Resultados mediante playLearning ${ }^{\circledR}$ : Valoración global de la prueba

\begin{tabular}{|l|l|}
\hline VALORACIÓN GLOBAL DE LA EXPERIENCIA PILOTO & $\mathbf{3 . 6 4}$ \\
\hline Utilidad: & 3.66 \\
\hline La actividad me ha aportado nuevos conocimientos y habilidades & 3.57 \\
\hline $\begin{array}{l}\text { El laboratorio, ¿ha potenciado el uso de la REI como herramienta } \\
\text { de trabajo? }\end{array}$ & \\
\hline Documentación: & 3.48 \\
\hline La información recibida al inicio de la experiencia ha sido adecuada & 3.26 \\
\hline Actividades: & 3.64 \\
\hline $\begin{array}{l}\text { La distribución del tiempo planteada (lectura, actividades...) ha sido } \\
\text { adecuada }\end{array}$ & \\
\hline $\begin{array}{l}\text { Las actividades me han ayudado a descubrir el potencial de la REI } \\
\text { como herramienta de trabajo }\end{array}$ & 3.40 \\
\hline Plataforma: & 3.08 \\
\hline $\begin{array}{l}\text { El entorno desarrollado ha sido fácil de manejar y su uso ha favorecido } \\
\text { el uso de la REI }\end{array}$ & 3.43 \\
\hline $\begin{array}{l}\text { A lo largo de la acción formativa NO me he encontrado problemas } \\
\text { técnicos que me hayan impedido la normal realización. }\end{array}$ & $\begin{array}{l}\text { Si he tenido problemas técnicos, me he sentido atendido y la resolución } \\
\text { ha sido rápida y satisfactoria }\end{array}$ \\
\hline
\end{tabular}

Fuente: Elaboración propia a partir de los datos obtenidos en la Red 


\section{Cuadro $n^{o}$ 4. Resultados: Seguimiento Tutorial}

\begin{tabular}{|l|l|}
\hline \multicolumn{2}{|l|}{ SEGUIMIENTO TUTORIAL } \\
\hline $\begin{array}{l}\text { En caso de cualquier duda relacionada con la metodología y organiza- } \\
\text { ción del curso, siempre he contado con la presencia y ayuda } \\
\text { del CTP }\end{array}$ & 3.74 \\
\hline $\begin{array}{l}\text { El CTP me ha dado todo el apoyo necesario en el curso y su tiempo de } \\
\text { respuesta, en caso de cualquier duda, ha sido breve (menos de 24 h.) }\end{array}$ & 3.74 \\
\hline Método de enseñanza: & \\
\hline La duración total del curso ha sido apropiada & 3.14 \\
\hline $\begin{array}{l}\text { La metodología propuesta ha sido adecuada para el fomento del uso } \\
\text { de la REI }\end{array}$ & 3.44 \\
\hline $\begin{array}{l}\text { La metodología playLearning® ha supuesto un valor añadido a la } \\
\text { utilidad habitual de la REI }\end{array}$ & 3.70 \\
\hline Compañeros: & 3.48 \\
\hline $\begin{array}{l}\text { La relación con sus compañeros del curso le ha supuesto algún } \\
\text { enriquecimiento }\end{array}$ & 3.50 \\
\hline $\begin{array}{l}\text { ¿Consideras enriquecedor el intercambio a tiempo real con tus compa- } \\
\text { neros del Laboratorio? }\end{array}$ & \\
\hline
\end{tabular}

Fuente: Elaboración propia a partir de los datos obtenidos en la Red

A continuación de las preguntas de respuesta cerrada, se incluyeron un conjunto de preguntas de respuesta abierta, campos de libre disposición que los usuarios pueden utilizar para hacer comentarios y sugerencias de mejora. De todas ellas, se pueden destacar por tener un número elevado de repeticiones los siguientes aspectos positivos:

- Mayor interacción entre personajes ofrecida por la plataforma.

- Comunicación síncrona.

- Nueva modalidad de formación, combinando el carácter social.

- Optimizar el manejo de la red.

- Facilidad de aprendizaje en forma de juego.

- Sencillez del formato.

- Facilidad de navegación.
Y los aspectos a mejorar:

- Lentitud de la carga.

- Ventanas de chat no trasladables a los distintos escenarios.

- Requisitos altos de software y hardware.

- Personalización de los avatares.

- Ausencia de aspectos lúdicos.

- Ampliar la actividad de los avatares.

De dichas respuestas de tipo abierto, incluimos a continuación un conjunto que refleja opiniones significativas de los usuarios:

Pregunta: ¿Consideras que este acceso a la REI debería ser permanente?

- Sí, por lo menos por un tiempo a modo de prueba, ya que agiliza el movimiento a través de la REI y de forma más didác- 
tica e interactiva. Además permite un contacto mayor con otros participantes.

- Considero que esta modalidad virtual es aplicable como acceso y entorno a la REI, eso permitirá estrechar los contactos de los miembros.

- Estoy de acuerdo, porque todo lo que incide en realizar el trabajo de mejor forma, tiempo, y mejores prácticas serán herramientas valiosas.

- Totalmente, el ambiente de interacción en la REI debería ser este permanentemente, eso motiva y promueve a los expertos a participar activamente de la REI.

- En efecto, así todos los que estamos dentro de la REI, tendríamos la opción de consultar, investigar periódicamente, todos los avances, novedades, etc., que se estén desarrollando.

Pregunta: ¿Lo harías extensible al resto de Redes de Expertos?

- Sí, lo haría extensible a otras redes, la difusión en esta propuesta es de una metodología innovadora que por su potencial y alcance merece ser puesta en práctica en otras áreas temáticas.

- Claro que sí (Los diversos profesionales, deben tener las nociones claras de a dónde vamos con la educación y los nuevos espacios de desarrollo económico - social, con el fin de tomarlos en cuenta como alternativas de desarrollo y difusión de sus diversos proyectos).
- Totalmente de acuerdo!! Sería un espacio integrador donde podamos interactuar con todas las ventajas que ofrece el mundo virtual.

Pregunta: ¿Añadirías algún elemento al entorno desarrollado?

- Sí sonido, cuando un compañero se comunica con uno y que la ventana del diálogo no desaparezca cuando se ingresa por ejemplo a los grupos, a la cafetería, etc. Facilitaría la continuidad del diálogo y del aprendizaje.

- Debe permitirse al avatar interactuar con otros elementos del entorno, como tomar agua o comer, sentarse, saludar, $\mathrm{u}$ otros que permitan dar apariencia real a lo virtual.

- La posibilidad de diseñar un avatar a gusto de uno, es decir que tenga características más parecidas al usuario.

- Me gustaría un espacio donde se puedan realizar videoconferencias.

- Un chat de voz sería mucho más enriquecedor.

- Si un "relojero" o personaje que controlara o indicara en QUÉ actividad estamos en la semana y DÓNDE la debemos cumplir (en lo que refiere a lo obligatorio).

Pregunta: ¿Consideras útil este acceso para otro tipo de aplicaciones?

- Considero la experiencia sumamente pertinente de ser extrapolada a otros entornos que, bajo diferentes objetivos 
de aprendizaje, requieran la ambientación de los participantes en contextos signados por la interdisciplinariedad y la multiculturalidad y el desarrollo de competencias - conocimientos, capacidades y actitudes - especialmente las personales y sociales.

- Este tipo de acceso es importante para todo tipo de aplicaciones, medicina, ingeniería, capacitaciones, estudios, negocios, juicios, debates, etc.

- Claro que sí, ejemplo en la lucha y prevención del blanqueo de capitales, propiciado por expertos para que sea certero. Propondría al Dr. Gonzalo Gómez De Leaño Polo, del Instituto de Estudios Fiscales del Ministerio de Hacienda de España, porque la experiencia que él posee es de altos kilates.

- Claro que sí, desarrollo de cursos de inducción a funcionarios nuevos en organizaciones, para desarrollar cultura y capacitación a ciudadanos en temáticas particulares, grupos de discusión y conocimientos técnicos.

Pregunta: Señale los aspectos del curso que le han parecido más positivos.

- Metodología de aprendizaje innovadora. Aplicación de la metodología de aprendizaje a diferentes propósitos relacionados con los ámbitos laborales y profesionales de los participantes. Interacción de los participantes desde su cultura, experiencia y ámbito de desempeño.
- Intercambio de conocimientos, Campus virtual innovador (plataforma), Asesoramiento profesional (tutorías).

- Romper paradigmas mentales de educación tradicional. El intercambio de comentarios entre un experto en la temática que sacó un valor agregado al curso de triple A.

- Aprendizaje sobre los mundos virtuales, la variedad de entornos, aplicación al mundo real y su contribución al futuro de la sociedad.

- El apoyo permanente del coordinador del curso, especialmente en la resolución de problemas técnicos o cuando el participante no está pudiendo llevar el laboratorio en los plazos establecidos.

- El hecho de mantener comunicación con los compañeros del curso en tiempo real y debatir sobre las actividades a realizar.

- Otro punto positivo fue poder crear temas para invitar a participar a los otros expertos y que dejen sus conocimientos sobre el tema.

Pregunta: Señale aquellos aspectos del curso que en su opinión deberían mejorarse.

- Los chats deberían permanecer abiertos cuando accedemos a las mesas de discusión, visto que cuando accedíamos a ellas los chats eran interrumpidos y sólo se podría volver a hablar en ellos al salir de la mesa. 
- Para empezar este laboratorio cumplió con las expectativas esperadas, lo que se podría mejorar es que quede abierto esta modalidad para todas las redes, de modo de tener más personajes con los que se puede interactuar, también fuera bueno crear un edificio del estudiante donde cada uno podría tener su propia habitación, en la cual podría colocar datos personales, fotos, videos, etc. Y así mismo poder visitar habitaciones de los compañeros.

- Plataforma lenta en cargarse.

- Falta de sincronización de encuentros prácticos de este tipo de espacios MUVE's (Problemas principalmente en los horarios de encuentros sincrónicos).

- Respecto a aspectos que deberían mejorarse, a mi parecer sería mejorar de una forma más accesible el traspaso de ventanas en las interfaces.

- Me parece que faltaría un poco más de coordinación y organización, específicamente en la designación de grupos. El tutor o coordinador debe de indicar directamente los integrantes del grupo y designar de ahí a un líder, para que de esta manera el trabajo en equipo sea más efectivo (designando tareas, actividades, comisiones, etc.).

Pregunta: Indique otros posibles temas sobre los que realizaría una nueva acción formativa.

- Específicos de gestión de residuos. La verdad que me gustaría alguno sobre
Sistemas Integrados de Gestión para diferentes topologías de residuos, compostaje, etc. Como Coordinador temático estaré proponiendo varios espacios de intercambio (foros) sobre estos temas (pilas usadas, biofertilizantes, bolsas plásticas, tecnología limpia, biodigestión, etc.).

- Catastros virtuales, Gestión del sector público, Administración del catastro, sistemas de información geográfica.

- Temas relacionados con la Gestión de Recursos Humanos, Psicología, Análisis de Entorno entre otros.

- EGoverment que abarque el gran conglomerado de recursos que está a su disposición y cómo este se articula, por ejemplo un mapa conceptual de su integración (eServices, eLearning, eComerce, eTrabajo, etc.); la globalización empuja a la obligatoriedad de uso de este tipo de recursos, pero cómo tener un Modelo Conceptual claro de su articulación y aplicación.

- Propiedad intelectual, Tecnología, Leyes.

- Me gustaría un curso que vincule y actualice sobre "el marketing y los mundos virtuales", nuevos usuarios, nuevas formas de informar, de promocionar, publicidades 3D y opiniones de expertos.

- Siento muchísima curiosidad porque profundicemos en temas como: Internet 2, Herramientas Web 2.0, Diseño 
de Mundos Virtuales dedicados al Aprendizaje, Uso de la Plataforma Moodle, Software desarrollador de ambientes virtuales.

- Metodología de la investigación, y gestión de la seguridad social y especialmente de los servicios sanitarios.

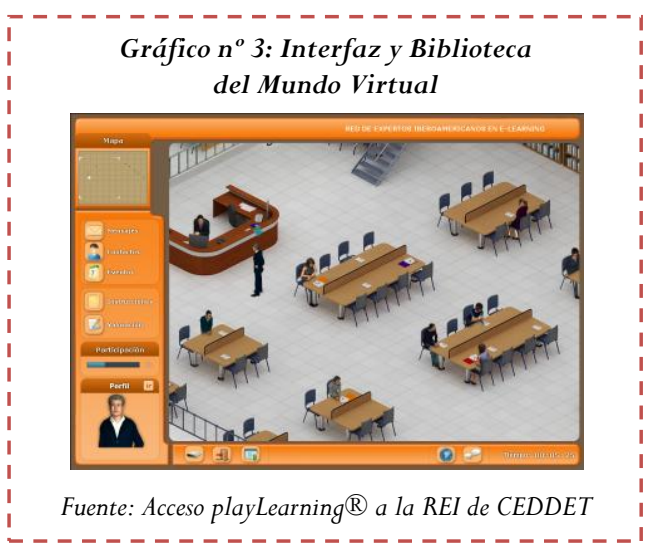

\subsection{Reflexión}

La investigación llevada a cabo permite hacer una breve reflexión sobre el impacto de las redes sociales corporativas, y sobre todo, sobre cómo éstas son implementadas en los entornos profesionales en las empresas.

En primer lugar, cabe destacar el estado incipiente de las redes corporativas en el mundo empresarial. Tal como apunta Tenzer (2009), la tendencia ha empezado a revertirse, y muchas empresas comienzan ya a beneficiarse de estas redes sociales.
Las ventajas del uso de las redes sociales son múltiples en el mundo empresarial. Tal como indica Ranera (2010), van desde fomentar la cultura de participación, compromiso y colaboración, potenciar la relación entre empleados y el orgullo de pertenencia, aumentar la implicación e innovación, detectar y retener el talento, preservar los valores de la compañía, mejorar la productividad y efectividad de los procesos, agilizar la comunicación y coordinación entre equipos, o aumentar la velocidad en la toma de decisiones. Además, el aprovechamiento de la red de contactos termina por repercutir positivamente en la gestión de los proyectos y de los clientes, lo que influye finalmente en la cuenta de resultados (Tenzer, 2009).

Hasta ahora las redes sociales, incluyendo las corporativas, se han basado mayoritariamente en los entornos web, fundamentalmente html, que con sus ventajas e inconvenientes es la tecnología más accesible. En nuestra investigación, partiendo de la base de las ventajas de las redes sociales corporativas, hemos llevado a cabo una comparación entre el acceso web tradicional, con el acceso mediante los mundos virtuales inmersivos, obteniendo un resultado altamente positivo que, tal como exponemos en las conclusiones, mejora la interacción y motivación de los usuarios en su manejo. 


\section{Conclusiones}

En base al análisis de los resultados obtenidos, podemos extraer las siguientes conclusiones:

- Se ha desarrollado el prototipo Red Social destinado a usuarios de la red sin conocimientos previos sobre el manejo del entorno.

- Se ha realizado una prueba con 100 alumnos reales en colaboración con la Fundación CEDDET, con el objetivo de demostrar y validar la metodología playLearning ${ }^{\circledR}$, de profundizar en su diseño y desarrollo, y como forma de obtener un feedback de los usuarios para poder mejorar la metodología y su desarrollo.

- Se han mejorado los procesos internos de desarrollo de la metodología.

- La prueba ha sido satisfactoria, obteniendo una gran variedad de comentarios por parte de los participantes, de los que se pueden extraer las principales vías de mejora para futuras versiones. En cuanto a la valoración cuantitativa, y poniéndolo en escala de 1 a 10 , se ha obtenido un valor de 9.1, que mejora el valor obtenido en el acceso web estándar de 8.1 .

- Se deben mejorar aspectos relacionados con la metodología, relacionados con la organización y las instrucciones que se ofrecen a los participantes.
El equipo que ha desarrollado e impartido la acción formativa, después de estudiar las valoraciones realizadas por los participantes, realiza las siguientes reflexiones y propuestas de mejora:

- Valorar la importancia de la comunicación previa de la acción formativa, mejora de las instrucciones de uso y manejo del entorno.

- Mejorar velocidad de carga y los requisitos mínimos para acceder al entorno.

- Mostrar los contenidos de los Foros en forma de árbol.

- Incluir la posibilidad de personalizar más los avatares.

- Incluir funcionalidades lúdicas y ampliar el movimiento de los personajes.

- Mantener la funcionalidad de chat en los distintos escenarios disponibles.

Como línea de investigación en el futuro, se desarrollará un mundo virtual para el entorno de la Tecnología Sanitaria. Este desarrollo permitirá poner en valor el proyecto descrito, profundizar y mejorar los aspectos relacionados con la metodología y con la organización, y además permitirá estudiar las implicaciones de carácter comercial, abriendo un nuevo conjunto de posibilidades de explotación de los mundos virtuales, en este caso orientados al sector sanitario. 
Como conclusión final, y volviendo a la pregunta:

\section{¿Los entornos inmersivos pueden mejorar la interacción y la motiva- ción de los usuarios en las redes cor- porativas?}

Podemos concluir, gracias al éxito de la experiencia piloto, que efectivamente los entornos inmersivos mejoran la interacción y la motivación de los usuarios en las redes corporativas. Está claro que tecnologías similares se irán imponiendo en el futuro sobre otras que, pese a su juventud, se pueden considerar ya "tradicionales".

\section{Referencias}

HERNÁNDEZ GARCÍA, ILIANA (2002): Mundos virtuales habitados: Espacios electrónicos interactivos. Ed. Universidad Javeriana.

BARTLE, RICHARD A. (2003): Designing Virtual Worlds. Ed. New Riders.

MARTÍNEZ LÓPEZ, RUTH (2010): Mundos virtuales 3D: Una guía para padres y formadores. $E d$. UOC.

CABERO ALMENARA, JULIO (2007): Diseño y Producción de TIC para la formación.Ed. UOC.

ASINSTEN, JUAN CARLOS (2007): Producción de contenidos para Educación Virtual. Guía de trabajo del docente-contenidista. Ed. Virtual Educa.

MONSORIU FLOR, MAR (2008): Manual de redes sociales en internet. Ed. Creaciones Copyright.

DE UGARTE, DAVID (2008): El poder de las redes. $W w W$.deugarte.com

MAYFIELD, ANTONY (2008): What is Social Media? icrossing.co.uk/ebooks

TENZER, SIMÓN M., FERRO, OLGA, PALACIOS, NURIA (2009): Redes sociales virtuales: Personas,sociedad y empresa.

http: //salonvirtual.upel.edu.ve/pluginfile.php /19830/mod_resource/content/O/Redes_Soci ales/Redes.pdf

RANERA, MIREIA (2010): Redes sociales corporativas, la asignatura pendiente en
España. http: / / www.mujeresconsejeras.com/redessociales-corporativas-la-asignatura-pendiente-enespana/2010/10/19/

EGGENS, LILIAN, VAN DER WERF, M.P.C.(GREETJE), BOSKER, ROEL J. (2007): The influence of personal networks and social support on study attainment of students in university education. Higher Education (2008) Vol. 55, No. 5: 553-573. Springer Science+Business Media B.V.

HEATH, SUE, FULLER, ALISON, PATON, KAREN (2008): Network-based ambivalence and educational decision-making: a case study of 'nonparticipation' in higher education. Research Papers in Education, Vol. 23, No. 2: 219-229.

LEE, MARK J.W., MILLER, CHARLYNN, NEWNHAM, LEON (2008): RSS and content syndication in higher education: subscribing to a new model of teaching and learning. Educational Media International, Vol. 45, No. 4: 311-322.

Routledge, Taylor \& Francis, Ltd. 


\section{NOTAS}

i "Red social corporativa: moda y necesidad". Entrevista a Enrique Aliende, Director General de IFO. Learning Review España n ${ }^{\circ}$ 7. http://www.learningreview.es/tecnologias-para-e-learning-09/1345-red-socialcorporativa-moda-y-necesidad

${ }^{i i}$ PlayLearning® es una metodología de eLearning desarrollada y registrada por Instituto de Formación Online S.L. en la que los usuarios aprenden practicando en un entorno de mundo virtual a través de un avatar que les representa, y deben ir alcanzando objetivos formativos que se van reportando a una plataforma de eLearning compatible SCORM.

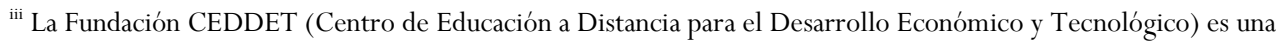
organización sin ánimo de lucro dedicada a la cooperación al desarrollo que fue creada a iniciativa del Ministerio de Economía y Hacienda de España y el Banco Mundial.

iv Galeno XXI es un proyecto enmarcado dentro del Plan AVANZA2, Subprograma Avanza Competitividad $(\mathrm{I}+\mathrm{D}+\mathrm{i})$, y sus objetivos fundamentales son la creación de una herramienta online bilingüe (español e inglés) para la formación de médicos de atención primaria, mediante contenidos en un entorno 3D de mundo virtual, bajo la metodología playLearning ${ }^{\circledR}$ y compatible con la iniciativa de estandarización SCORM. 